Thorax 1987;42:77-78

Short report

\title{
Massive haemoptysis as a late consequence of pulmonary irradiation
}

\author{
R D ISAACS, W J WATTIE, A U WELLS, H H REA, T R BAI
}

From the Departments of Radiology and Respiratory Medicine, Green Lane Hospital, Auckland, New Zealand

\begin{abstract}
Massive haemoptysis has not been reported as a late complication of therapeutic pulmonary irradiation. We report two cases of massive haemoptysis arising from abnormal bronchial arteries in lung parenchyma previously exposed to therapeutic doses of radiation.
\end{abstract}

\section{Case reports}

\section{PATIENT 1}

A non-smoking caucasian woman presented in 1978, aged 23 years, with a stage IIB rhabdomyosarcoma of the right thigh. She was treated with local irradiation and intravenous chemotherapy with vincristine, actinomycin D, and cyclophosphamide. Three months later a pulmonary nodule assumed to be a metastasis was noted on the chest radiograph in the right lower lobe. Radiotherapy using a cobalt teletherapy unit delivering a total tumour dose of $45 \mathrm{~Gy}$ $(4500 \mathrm{rad})$, in 15 fractions, into a treatment volume of $4 \times$ $4 \mathrm{~cm}$ resulted in disappearance of the nodule. Monthly chemotherapy with adriamycin, cyclophosphamide, vincristine, and dacarbazine was given for 10 months.

Over the next five years there was no evidence of tumour recurrence. She presented with haemoptysis totalling $600 \mathrm{ml}$ in 1984. There was no evidence of infection. Scarring in the region of the right hilum was seen on the chest radiograph and computed tomography scan. Profuse bleeding arising from the right lower lobe was seen at bronchoscopy. Bronchial arteriography identified abnormal vessels supplied by the right lower bronchial artery in the region of the radiotherapy scar (fig 1). Bronchial artery embolisation with Ivalon particles (polyvinyl alcohol, Unipoint Industries, North Carolina) and a very slow infusion of absolute alcohol was performed under fluoroscopic control. At follow up bronchoscopy showed nothing abnormal and an arteriogram demonstrated occlusion of the right lower bronchial artery. A pulmonary arteriogram was unremarkable. Sixteen months later she remains well.

\section{PATIENT 2}

A caucasian boy presented at the age of 16 months in 1969 with cervical adenopathy and a mediastinal mass. Biopsy specimens initially suggested lymphoma. He received a dose of $10 \mathrm{~Gy}(1000 \mathrm{rad})$ from a cobalt teletherapy unit to the neck and mediastinum, in seven fractions over 10 days. Review of the histological specimens changed the diagnosis to benign sinus histiocytosis. ${ }^{1}$ He remained well until 1975 , when he underwent total thyroidectomy for papillary thy-

Address for reprint requests: Dr T R Bai, Department of Respiratory Medicine, Green Lane Hospital, Auckland, New Zealand.

Accepted 16 May 1986 roid carcinoma; $50 \mathrm{mCi}$ of iodine 131 was given postoperatively. His progress over the next five years was uncomplicated.

In 1980 he presented with minor haemoptysis. Bronchoscopy showed only prominent submucosal vessels in the trachea. In 1985 he presented with repeated major haemoptyses, totalling $1500 \mathrm{ml}$ over eight days. There was no evidence of infection. A chest radiograph showed changes in the left mid zone consistent with bleeding within the lung. Bronchoscopy showed blood throughout the left tree. A bronchial arteriogram showed a dilated left artery, which divided close to its origin into upper and lower lobe branches. Both vessels were tortuous and dilated and areas of hypervascularity were clearly defined throughout the left lung (fig 2). Ivalon embolisation of the lower artery was followed by Gelfoam (absorbable gelatin powder, Upjohn

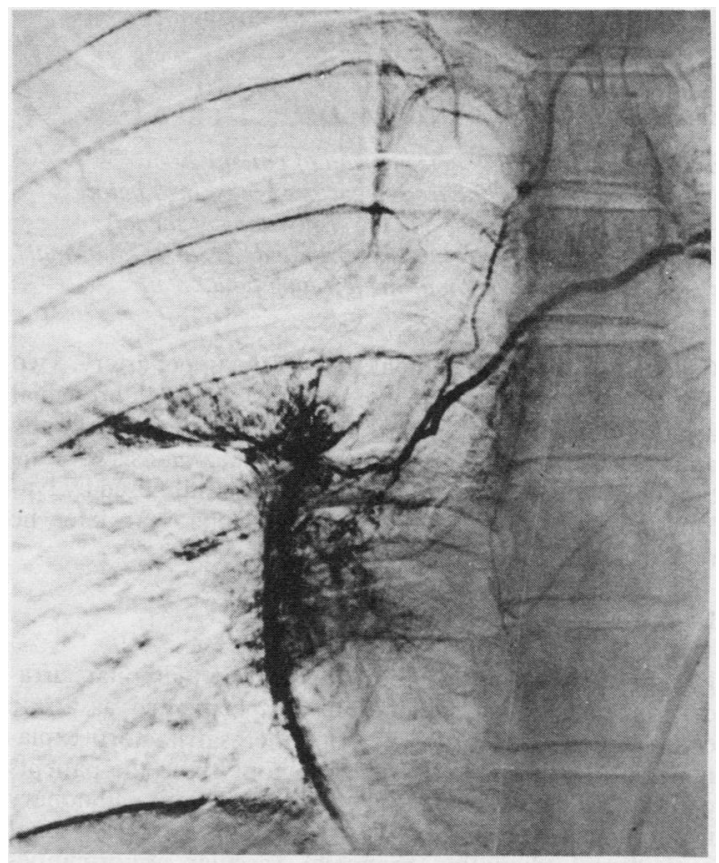

Fig 1 Right bronchial arteriogram of patient 1 (conventional film subtraction) before embolisation. Tiny vessels, some running lateral to the hilum and others descending parallel to the right heart border, are shown in an area distorted by the scar from previous radiotherapy. $A$ group of small, dilated arteries is defined medial to the scar. 


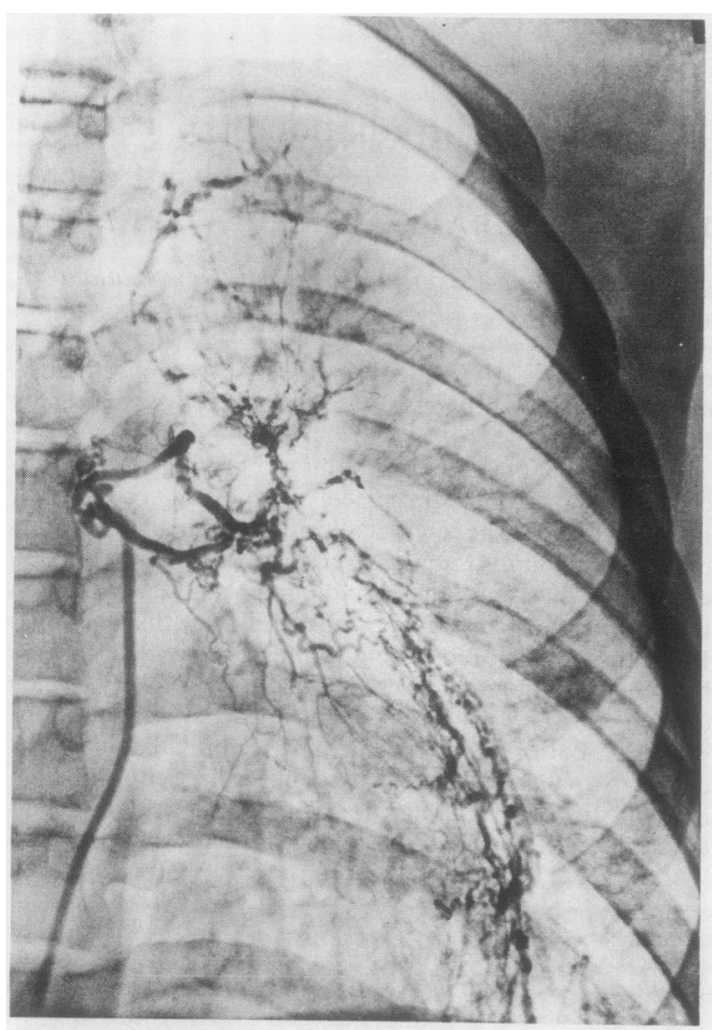

Fig 2 Left bronchial arteriogram of patient 2 (photographically enhanced by unsharp masking) before embolisation. The upper and lower divisions of the left bronchial artery are tortuous and dilated. Areas of abnormal vessels are seen throughout the left lung field.

Company, Michigan) embolisation of the upper artery. Two days later, after a further haemoptysis of $200 \mathrm{ml}$, a bronchial arteriogram showed the upper artery to be patent proximally and the pulmonary artery filling via an anastomosis with the bronchial artery. The bronchial artery was embolised by Ivalon, Gelfoam, and absolute alcohol. One year later he remains well.

\section{Discussion}

Despite the widespread use of therapeutic pulmonary irradiation, haemoptysis has not been documented as a late complication. In the absence of other satisfactory explanations, it is probable that the haemoptysis in the patients described in this report resulted from previous pulmonary irradiation. It is possible, although unlikely, that coincidental idiopathic bronchial vascular abnormalities existed in these two patients. There was no evidence of suppurative lung disease or primary or secondary lung neoplasm in either case. Abnormal bronchial arteries were seen within the radiation field in both cases. Prompt contro of haemoptysis by embolisation strongly indicated that these abnormal vessels were the source of the bleeding.

The pathogenesis of the vascular abnormalities is unclear Despite extensive documentation of toxicity from pul monary irradiation in man and animals, ${ }^{2}$ the changes wब describe have not been reported, although similar hypero vascularity has been observed by us in the rectal vasculature after local irradiation. Previous investigators have concen $\overrightarrow{0}$ trated on the pulmonary circulation, but bronchiaiarteriographic studies have not been documented.

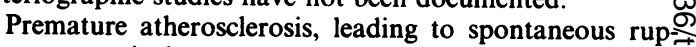
ture, occurs in large vessels exposed to radiation. ${ }^{3}$ Endo $\vec{x}$ thelial cells in the lung are susceptible to radiation injury ${ }^{2}$ new capillary formation has been demonstrated in histologi cal studies in rats. ${ }^{4}$ Ivashchenko ${ }^{5}$ has shown in a rabbit model that pulmonary irradiation results in the dilatation of major vessels, the opening of arteriovenous anastomoses $\frac{\text { O }}{2}$ and the deformation of small vessels. Endothelial damagefrom irradiation seems likely, at least in part, to be responsible for the changes we report. The chemotherapy given to Patient 1 may have enhanced the toxicity of pul-
monary irradiation. ${ }^{6}$

Alternatively, the hypervascularity in our patients may be $\vec{\circ}$ a non-specific response to pulmonary injury. Similaro changes in the bronchial arteries have been demonstrated in pulmonary infections ${ }^{7}$; Charan et $\mathrm{al}^{8}{ }^{8}$ using a sheep model of lung abscess, have suggested that the bronchial circus lation is more important than the pulmonary circulation in resolution and repair after lung infections.

Radiation induced bronchial artery damage should be⿳亠े⿵冂卄 considered in the differential diagnosis of haemoptysis. Embolisation at the time of bronchial arteriography wasō successful in both cases.

We thank Dr C B Mahood, respiratory physician, Waikato Hospital, for permission to report patient 1 .

\section{References}

1 Becroft DMO, Dix MR, Gillman JC, MacGregor BJL, Shaw RL. ̊. Benign sinus histiocytosis with massive lymphadenopathy: transient immunological defects in a child with mediastinalo involvement. J Clin Pathol 1973;26:463-9.

2 Gross NJ. The pathogenesis of radiation-induced lung damage. Lung 1981:159:115-25.

3 McCready RA, Hyde GL, Birins BA, Mattinghy SS, Griffin WO. Radiation-induced arterial injuries. Surgery 1983;93:306-12. ON

4 Maisin JR, Reyners H, de Reyners EG. Changes in the ultra- $N$ structure and the permeability of the capillaries after irradiation. Bibl Anat 1977;15:311-4.

5 Ivashchenko AP. Angiographic studies of the lungs after localo $x$-irradiation. Med Radiol (Mosk) 1967;12:31-8.

6 Phillips TL, Wharam MD, Margolis LW. Modification of radi- ation injury to normal tissue by chemotherapeutic agents. Cancer 1975;35:1678-84.

7 Cudkowicz $L$. The human bronchial circulation in health and disease. Baltimore: Williams and Wilkins, 1968.

8 Charan NB, Turk GM, Dhand R. The role of bronchial circu- $-\frac{1}{\mathbb{D}}$ lation in lung abcess. Am Rev Respir Dis 1985;131:121-4. 